\title{
Searching for Evidence Regarding Using Preoperative Disinfection showers to Prevent Surgical Site Infections:
}

A systematic review

\author{
Jakobsson J, RN, McS ${ }^{1}$, Perlkvist A, RN ${ }^{1}$, Wann-Hansson C RN, $\mathrm{PhD}^{2}$
}

${ }^{1}$ Department of Surgery, Malmö University Hospital, Entrance 42, 20502 Malmö, Sweden, T: +46 04 336947, jenny.a.jakobsson@skane.se, agnetha.perlkvist@skane.se

${ }^{2}$ Faculty of Health and Society, Malmö University, Entrance 49, 20506 Malmö, Sweden

T: +46406657430 fax: +46 40 6658100, christine.wann-hansson@mah.se 


\begin{abstract}
Background: Postoperative surgical site infections (SSI) are the third most common healthcare-associated infection. Even though several studies have pointed out the benefits from skin disinfection prior to surgery in order to reduce SSI, it remains unclear how to optimize the skin disinfection procedure.
\end{abstract}

Aim: The aim was to find evidence for how many times preoperative disinfection showers should be performed in order to reduce bacterial colonies and minimize the risk of SSI.

Method: A comprehensive literature search of multiple databases published from 1986 to 2008, supplemented by a manual search of the references in all relevant articles. Protocols were used in quality assessment and the data synthesis is descriptive in a narrative form. Results: The ten studies included had different designs, interventions, and samples, which makes it difficult to compare them. Moreover, the quality of the reviewed studies varied and only four had a high level of evidence. Therefore the results failed to give an unambiguous answer about the optimal number of preoperative showers, so only assumptions can be made. It is quite obvious, however, that preoperative disinfection showers with Chlorhexidine gluconate (CHG) are effective from a microbiological point of view since eight of the reviewed studies showed a sharply reduced skin flora after using CHG.

Conclusions: Currently, clear evidence for how many times preoperative disinfection showers should be performed to minimise the risk of SSI is missing. This highlights the need for further research that focuses on the number of preoperative disinfection showers in relation to SSI, in order to obtain optimal effect. Until then it would be wise to follow previously made recommendation of 3-5 preoperative showers. Moreover, in order to have the intended effect of preoperative disinfection, it is important that healthcare professionals have the knowledge to guide patients with information and clear instructions of the disinfection shower procedures. 
Keywords: preoperative showers/baths/care, skin disinfection, transient skin flora, Chlorhexidine gluconate, postoperative surgical site infections 


\section{BACKGROUND}

Infections go back to the beginning of life itself and have always been a problem in our health care systems. Healthcare associated infections are still one of the most common complications, leading to longer hospital stays, increased costs and, not least, suffering for the patients which cannot be calculated in costs. Postoperative surgical site infections (SSI) are, after urinary tract infections and pneumonia, the third most common healthcare associated infection (Emori et al. 1993; Burke 2003; Gravel et al. 2007). Studies have shown that patients who acquire SSI double the length of their hospital stays and the risk of death (Dellinger 2005). In Sweden, the Swedish National Board of Health and Welfare (Socialstyrelsen 2006) strongly recommends patients who are about to undergo surgery where skin bacteria can cause serious infections, to prepare themselves by showering with CHG 3-5 times before surgery. Even though several studies have pointed out the benefits from skin disinfection prior to surgery (Bröte et al. 1976; Zandi 2000; Segal 2006) it remains unclear how to optimise the skin disinfection procedure. Evidence-based information is therefore needed that can guide healthcare professionals in making appropriate decisions regarding preoperative skin preparation procedures. Preoperative skin preparation is a process involving both preoperative shower/bath as well as the disinfection of the surgical site immediate prior to surgery. Current study focus only on preoperative shower/bath.

Already during the 1850’s, Florence Nightingale was a pioneer and reformer of hospital sanitation methods, although she was unaware that bacteria are the cause of infections (LaForce 1993). In the late 1860’s Lister introduced the concept of asepsis into the practice of surgery and started to disinfect skin, suture materials, and wounds, leading to a reduction in mortality after amputations. These actions later became supported by the discovery made by Pasteur of bacteria and their impact of putrefaction (Lidwell 1987). During the $20^{\text {th }}$ century 
the aseptic technique was developed and decreased the rate of infections, improving possibilities in surgery. However, despite the progress of medical treatment, such as introduction of chemotherapy and antibiotics, healthcare-associated infections still frequently occur. Large European multicentre studies have shown an overall prevalence of healthcare associated infections of 4-10\% (Harbarth et al. 1999; Gravel et al. 2007).

The resident skin flora exists both on the skin surface and deep skin layers. Twenty percent of the skin flora consists of resident bacteria and cannot be removed by washing or skin disinfection (Selwyn et al. 1972). The transient skin flora can be found in the mouth and nose, as well as in wounds, and is easily reduced by washing or skin disinfection (Tammelin et al. 2003; Nielsen et al. 1975). Chlorhexidine gluconate (CHG) used in antiseptic sponges is a skin disinfection product, which reduces the risk of microbial contamination. CHG attaches to the skin and has a prolonged effect, which can be an advantage during long-lasting surgery (Edmiston et al. 2007; Newsom et al. 1988; Mangram et al. 1999; Ayliffe et al. 1988; Lowbury et al. 1974). It has been demonstrated that the numbers of bacteria do not return until five days after disinfection with CHG (Lilly et al. 1979). By then, the surgical incision is normally healed (Newsom and Rowland 1988; Lindholm 2003). Further, it has been demonstrated that CHG has a cumulative effect if used repeatedly, and Lilly et al. (1979) showed a 99 \% reduction of the skin flora after five hand disinfections with CHG. Then there was a sharp rise in the numbers of bacteria, which was explained as bacteria present in deeper skin-layers being brought to the surface by the massaging effects of handwashing (Lilly et al. 1979).

Several studies have pointed out the benefit of preoperative disinfection and Zandi (2000) found no postoperative SSI in 800 patients who underwent two skin disinfections prior to 
liposuction surgery. However, diverse results are represented in the literature, and Segal (2006) argued that although preoperative showers reduce infection-causing bacterial colonies they cannot be directly associated with a reduction in SSI. Further, a Cochrane review aimed at find evidence for preoperative showering with antiseptics preventing SSI concludes that patients who performed a single preoperative shower had a higher rate of SSI than patients who had more than one preoperative shower. However, no clear evidence of benefits of preoperative showering or bathing with CHG over other wash products in order to reduce surgical site infection was found (Webster and Osborne 2009). Another review article, investigating the effect of disinfecting the specific site of surgery immediately before incision, found insufficient evidence to support or refute the use of skin disinfection at operative sites (Edwards et al. 2004). None of these studies aimed to investigate the most effective number of preoperative showers to prevent SSI. Moreover, the contrary results found in the literature justify a systematic review to guide practice in this area.

\section{AIM}

This study aimed to find evidence for how many times preoperative disinfection showers should be performed in order to reduce bacterial colonies and minimise the risk of postoperative surgical site infections.

\section{METHOD}

\section{Study selection}

In the process of specifying the assessment problem and determining inclusion criteria a structure presented by Flemming (1998) (Table 1) was used and the question at issue was "what is the most effective number of preoperative disinfection showers to reduce bacterial colonies and prevent surgical site infections?” This review explored the outcomes of different 
numbers of preoperative showers and different antiseptic solutions in studies with randomized or non-randomized experimental design. All categories of sample were included, from patients to healthy individuals, as well as a variety of healthcare-settings. Studies were included if the outcome was measured in relation to preventing surgical site infections or reducing skin bacteria flora. The systematic procedure for literature search and evidence interpretation described by the Swedish Council on Technology Assessment in Health Care (SBU 1993) was used as guidance.

\section{Literature search}

The literature search was carried out during 2007/2008 using standard methods and covered databases such as PubMed, CINAHL, and Cochrane Library. Initially, an unstructured literature search was carried out in PubMed. This search provided not only a hint of the amount of literature, but also guidance on the construction of inclusion- and exclusion criteria. The inclusion criteria were: articles about preoperative disinfection of the skin, published 1986 or later, elective surgery, randomised controlled studies, and clinical trials. Exclusion criteria were: articles about preoperative disinfection of hands or materials, published before 1986, languages other than English, and articles without an abstract.

The search strategy in each database included terms covering the supposed content and different types of study designs of interest combined with AND or OR. Search terms were “preoperative bathing”, “preoperative care” (MeSH), and “baths”. Every abstract was read and selection based on the inclusion- and exclusion criteria was made. The first search was conducted in PubMed with the search term "preoperative bathing” and resulted in forty-three articles. However, after further limitations according to the inclusion- and exclusion criteria, only 12 articles remained, nine of which were found to be relevant after reading the abstracts. 
The second search in PubMed, using the MeSH term "preoperative care”, resulted in 3,154 articles. When combining “preoperative care” AND “baths”, seven articles remained, all of which had already been found in the first search. The third search was conducted in CINAHL with the same terms and resulted in three articles, none of them matching the inclusion- and exclusion criteria. A fourth search was then performed, using the Cochrane database, that resulted in ten articles; but only three of these matched the inclusion- and exclusion criteria, and these had already been found in previous searches. Finally, a manual search was conducted by studying references from a research summary made by the Swedish National Board of Health and Welfare (Socialstyrelsen 2006), which resulted in one relevant study. In total, ten articles were found suitable for further review.

\section{Quality assessment}

All articles were read and revised by the first (JM) and second (AP) authors. A protocol made by Willman and Stoltz (2006) was used with the goal of critically reviewing the ten selected articles. The protocol focused on description and validation of study-design, intervention, and clinical value. The criteria for study quality and level of evidence were established according to SBU (SBU.se) (table 2).

After reviewing the articles individually, the results were compared and a final level of evidence of each study was established. Based on the level of evidence for each study, results were gathered and the grades of scientific foundations were established following SBU (SBU.se) (table 3).

\section{RESULT}


The ten articles included (Table 4) regarding the most effective number of preoperative showers were published between 1987 and 2008. After reviewing the studies, no conclusion could be established, due to the different overall designs. Byrne et al. (1990) was a pilot study with a small sample of healthy individuals. Eight studies used randomising procedures into different numbers of groups (Byrne et al. 1991; Earnshaw et al. 1989; Edmiston et al. 2008; Garibaldi 1988; Hayek et al. 1987; Kaiser et al. 1988; Rotter et al. 1988; Wihlborg 1987) and finally one study had a prospective follow-up design (Paulson 1993). Four studies investigated the differences between diverse numbers of preoperative showers (Byrne et al. 1990; Kaiser et al. 1988; Paulson 1993; Wihlborg 1987). Only one of these studies gained a medium level of evidence (Wihlborg 1987). Therefore the four studies together offer insufficient scientific foundation. Six studies described the superior effect of chlorhexidine on bacteria in comparison to other antiseptic solutions (mainly Povidone-Iodine and soap) and their focus was not on finding the most effective number of preoperative showers in order to minimise the risk of SSI (Byrne et al. 1991; Earnshaw et al. 1989; Edmiston et al. 2008; Garibaldi 1988; Hayek et al. 1987; Rotter et al. 1988).

\section{One preoperative shower}

Wihlborg (1987) included 1,530 surgical patients, randomised into three different groups. The first group washed the entire body with two consecutive applications of chlorhexidine on the day before surgery. The second group washed only the area of the body submitted to surgery, using chlorhexidine. The third group did not wash at all. The study gained a medium level of evidence and showed that one preoperative shower, with two consecutive applications of chlorhexidine, resulted in fewer SSI compared to no preoperative shower $(\mathrm{p}>0,01)$. A disinfection of only the incision area proved to be insufficient in order to escape contamination of bacteria from the body. Using a preoperative shower resulted in significantly 
fewer SSI $(p<0,05)$ compared to disinfection of only the area of incision. Garibaldi (1987) randomized 700 patients into four different groups preoperatively showering with chlorhexidine, povidone-iodine, soap, or not showering at all. Seven percent of the patients, who had no shower preoperatively, showed a contaminated wound intraoperatively, in comparison with $4 \%$ of the patients who showered with chlorhexidine preoperatively. No statistically valid comparisons in infection rates among the study groups were made because of the small study population.

\section{Two preoperative showers}

Kaiser et al. (1988) demonstrated that two preoperative showers resulted in a significant reduction of staphylococcal colonies $(\mathrm{p}<0.005)$ in comparison to one shower. However, this study gained a limited level of evidence because of the small sample of 36 subjects divided into six different groups. In another non-randomised study, involving 2,015 subjects, patients showered twice preoperatively with chlorhexidine, soap, or with a placebo. The number of infections among the patients showering with chlorhexidine was $9 \%$ compared to $12.8 \%$ in the soap group and $11.7 \%$ in the placebo group $(\mathrm{p}<0,05)$ (Hayek et al. 1987). The study of Edmiston et al. (2008) compared skin surface concentrations of CHG achieved after showering with 4\% CHG soap or cleansing the skin surface with an innovative 2\% CHGimpregnated polyester cloth. Mean CHG skin surface concentration were significantly higher in the group with two 4\% CHG showers (morning and evening) compared to levels observed in the group with only one 4\% HCG shower (evening). On the other hand there was no statistical difference noted in CHG skin concentration in patients who showered once in the morning and the group with two showers. These studies (Hayek et al. 1987; Edmiston et al. 2008) reached a high level of evidence. 
A randomized, double-blind multicenter study including 2,813 subjects investigated the use of two preoperative showers with chlorhexidine or a placebo in order to prevent SSI (Rotter et al. 1988). The study contained a well-described method and was valued as having high level of evidence. However, the results showed no significant difference between the incidence of postoperative SSI after preoperative showering, either in the chlorhexidine or placebo group $(\mathrm{p}>0,1)$

In a study with a moderate level of evidence Earnshaw et al. (1989) randomised two groups of patients ( $n=64$ and $n=66$ respectively) who underwent revascularisation. The first group used chlorhexidine and the second group used soap when showering preoperatively. The results could not prove the benefits from two preoperative showers either with chlorhexidine or soap. On the contrary, this study showed that the frequency of infections was twice as high in the chlorhexidine group as in the group using soap, though the difference was not significant.

\section{Three preoperative showers or more}

A significant reduction of the skin flora $(\mathrm{p}<0,01)$ after three preoperative chlorhexidine showers was presented by Byrne et al. (1991) in a study with moderate level of evidence. The study was randomised and blinded and after five days the patients were recolonied. Byrne et al. (1990) also tried to confirm the optimal number of CHG showers in a prior pilot study. Ten young and healthy study objects, aged 24-35, performed CHG showers, six times during six days. The greatest reduction of skin flora was demonstrated after two showers, and then the reduction was decreased after the third and fourth showers. On the other hand, the reduction increased again after the fifth shower. A significant skin flora reduction of $77.49 \%(\mathrm{p}<0,005)$ was seen after the second shower. Despite this, the authors finally did recommend three 
showers preoperatively because of the possibility that the patients do not perform a wash thoroughly enough (Byrne et al. 1990).

Paulson (1993) let five healthy individuals, aged 18-70 years, shower preoperatively with chlorhexidine during five days. Skin sampling, using agar plates, was conducted before the first shower, within ten minutes, and three and six hours after showering on days one, two, and five. The results showed a significant reduction of bacteria ten minutes after the first shower $(\mathrm{p}<0,05)$, which remained after three and six hours. The study also showed a cumulative effect in the reduced numbers of bacteria every day. This study gained only a limited level of evidence due to the small sample size.

\section{Discovery of notice}

The study by Kaiser et al. (1988) demonstrated interesting results when different types of antiseptics were compared, finding that soap increased the amount of bacteria cumulatively. Kaiser explained this paradoxical effect of using soap as being caused by those areas with fewer bacteria becoming contaminated by bacteria from other areas of the body containing more bacteria, for example the groin. This conclusion is supported by two other studies (Garibaldi 1987; Hayek et al. 1987).

\section{DISCUSSION}

This systematic review aimed to bring clarity on how many times preoperative disinfection showers should be performed in order to prevent SSI. The studies included all had different designs, however, making it difficult to compare them with each other. Moreover, the quality of the ten reviewed studies varied and only four had a high level of evidence. Limitations such as small samples and/or methodology shortcomings were reasons for being valued as having a 
limited level of evidence. In a majority of the reviewed studies, the number of disinfection showers was treated as a secondary matter and reasons why the particular number of showers was chosen were not further explained (Byrne et al. 1991; Edmiston et al. 2008; Earnshaw et al. 1989; Garibaldi 1988; Hayek et al. 1987; Rotter et al. 1988; Wihlborg 1987). Only four studies investigated the outcome of different numbers of preoperative showers (Byrne et al. 1990; Kaiser et al. 1988; Paulson 1993; Wihlborg, 1987) and only one of these was considered to have a medium level of evidence. The other three studies had limited evidence value which suggests that the scientific foundation is limited and the strengths of evidence concerning grades of recommendations are weak. Therefore the results failed to give an unambiguous answer about the optimal number of preoperative showers, and only assumptions can be made. However, it is quite obvious that preoperative disinfection showers with CHG constitute an effective skin disinfectant from a microbiological point of view since eight of the reviewed studies showed a sharply reduced skin flora after use of CHG products (Byrne et al. 1990; Edmiston et al. 2008; Kaiser et al. 1988; Wihlborg 1987; Paulsson 1993; Garibaldi 1988; Hayek et al. 1987; Byrne et al. 1990). It is apparent that further research focusing on the optimal number of preoperative skin disinfection procedures with multicenter randomised controlled designs is needed.

A cumulative effect of repeated showers with chlorhexidine was found in four of the reviewed studies (Byrne et al. 1990; Edmiston et al. 2008; Kaiser et al. 1988; Paulson 1993). However, the skin disinfection procedures were not always explicitly described and it was difficult to identify whether "a shower" meant a repeated or single shower. Only one of the reviewed studies confirmed that failure to implement a standardised, validated strategy for preoperative showering will likely result in potential gaps in effective CHG concentrations on the surface of the skin (Edmiston et al. 2008). According to recommendations from The Swedish 
National Board of Health and Welfare (Socialstyrelsen 2006), three preoperative repeated disinfection showers should be performed. This means to apply CHG soap to the whole body including the hair, protecting eyes and mucosa, and that after rinsing the procedure should be repeated. After each application, the 4\% CHG should remain on the skin surface for up to six minutes. If two showers in the reviewed studies meant the same as one repeated shower described above one assumption could be that the Swedish recommendations actually are comparable to six single showers and that patients today are invited to shower twice as many times as necessary. This could neither be considered to be cost-effective nor friendly to the skin. According to the Guideline for Prevention of Surgical Site Infection (Mangram et al. 1999) it is important that healthcare organizations implement policies to prevent transmission of microorganisms from personnel to patients and the document is primarily intended for use by surgeons and all other personnel directly responsible for the prevention of nosocomial infections. Besides the importance of compliance regarding guidelines for basal hygiene routines among healthcare professional's clinical practical guidelines for preoperative skin disinfection should also include instructions that are easy to understand and follow for the patients. Further, in order to be able to compare results and draw valid conclusions future research must also include well-described disinfection procedures.

Several conflicting results were shown in the reviewed studies. One of the studies advocates two preoperative showers by using the study subject as their own controls (Byrne et al. 1990), while two other studies were unable to prove benefits from showering twice when comparing chlorhexidine with placebo (Rotter et al. 1988, Earnshaw et al. 1989). One reason why no significant difference was obtained between the chlorhexidine and placebo group may depend on the patient characteristics. For example, the sample in the study of Rotter et al. (1988) consisted of patients who only underwent "clean surgery", where the incidence of SSI is 
considered to be marginal. Thus, no significant difference could be expected. On the other hand, the sample in Earnshaw et al. (1989) study consisted of vascular high-risk patients, e.g. those suffering from distal derma necrosis and it is well known that the risk of SSI is potentially higher in patients with contaminated wounds (Mangram et al 1999). It is also wellknown that that the incidence of postoperative wound infection depends on several risk factors such as age, antibiotic prophylaxes, time of operation, malignancy etc (Wihlborg 1987) and that to completely eliminate SSI is a complex problem depending not solely on the number of preoperative showers. The external validity could therefore be debated as it somewhat doubtful whether results from the different studies can be generalised to all kind of surgery patients.

One striking finding to consider was that one of the reviewed articles revealed that soap sharply increased the amount of bacteria, with a cumulative effect (Kaiser et al. 1988). This negative effect of soap was also supported by two other studies (Garibaldi 1987, Hayek et al. 1987). On the other hand, Earnshaw et al. (1989) demonstrated higher frequencies of infections among patients washing with chlorhexidine in comparison with patients using soap. The difference was however, not significant. Thus, a possible recommendation based on the findings of the three above studies would be to avoid showers with soap before surgery. Another recommendation according Edmiston et al. (2008) is that the use of 2\% CHGimpregnated cloths should be preferable over using 4\% CHG soap since the design of the polyester cloth was likely more efficient, allowing greater penetration of the active agent into the deeper recesses of the skin. However, the use of both soap and of $2 \%$ CHG cloths needs to be further evaluated before any recommendation based on a scientific foundation can be made. 


\section{Limitations of the review}

One limitation of this review was that only English language studies were included in the search strategies, which may have led to the omission of some relevant published articles. Another limitation was that merging the results was not possible due to the diverse designs and interventions in the reviewed studies and therefore the authors could provide only a narrative description of the ten reviewed studies. The difficulty in finding literature that focuses on the number of preoperative disinfection showers was also reflected in the review by Webster and Osborne (2009) which states that there were inconsistencies in both interventions and control procedures between studies. But since the purpose of the Webster and Osborne (2009) review was to examine the preoperative showers effect on SSI, the number of showers was mentioned only briefly.

The intention of the present study was to find more recent studies than The Swedish National Board of Health and Welfare (Socialstyrelsen 2006) are using as a basis for their recommendations; therefore one of the inclusion criteria was articles printed in 1985 or later. However, during the literature search it became obvious that research regarding preoperative skin disinfection has mostly focused on effects from different disinfection products and not the optimal use of these products. The reason that only a few recent studies were found may be that this is considered as well-understood area not needing any further exploration since it has been known since the late $19^{\text {th }}$ century that disinfection is important in healthcare (Lidwell 1987).

\section{CONCLUSION}

Currently clear evidence for how many times preoperative disinfection showers should be performed in order to minimise the risk of postoperative surgical site infections is missing. This highlights the need for further research that focuses on the number of preoperative 
disinfection showers in relation to SSI in order to obtain optimal effect. Until then, however, it would be wise to follow the recommendation of 3-5 preoperative showers made by The Swedish National Board of Health and Welfare (Socialstyrelsen 2006). Moreover, in order to have the intended effect of preoperative disinfection, it is very important that healthcare professionals have this knowledge in order to guide patients with information and clear instructions of the disinfection shower procedures.

\section{REFERENCES}

Ayliffe G A, Babb J R, Davies J G, Lilly H A (1988). Hand disinfection: a comparison of various agents in laboratory and ward studies. Journal of Hospital Infections, 11(3), 226-243.

Bröte L, Gillquist J, Tärnvik A (1976). Wound infections in general surgery. Wound contamination, rates of infection and some consequences. Acta Chirurgica Scandinavia, 142 (2), 99-106.

Burke J (2003). Infection Control. A Problem for Patient Safety. New England Journal of Medicine, 348 (7), 651-656

Byrne D J, Napier A, Cuschieri A (1990). Rationalizing whole body disinfection. Journal of Hospital Infection, 15(2),183-187.

Byrne D J, Napier A, Phillips G, Cuschieri A (1991). Effects of whole body disinfection on skin flora in patients undergoing elective surgery. Journal of Hospital Infection, 17(3), 217222.

Dellinger E P (2005). Increasing inspired oxygen to decrease surgical site infection. Journal of the American Medical Association, 249(16), 2091-2092.

Earnshaw J J, Berridge R C, Slack R C B, Makin G S, Hopkinson B R (1989). Do preoperative chlorhexidine baths reduce the risk of infection after vascular reconstruction? European Journal of Vascular Surgery, 3, 323-326. 
Edmiston CE, Seabrook GR, Johnson CJ (2007). Comparative of a new and innovative 2\% chlorhexidine gluconate-impregnated cloth with $4 \%$ chlorhexidine gluconate as topical antiseptic for preparation of the skin prior to surgery. American Journal of Infection Control, 35, 89-96.

Edmiston CE, Krepel CJ, Seabrook GR, Lewis BD, Brown KR, Towne JB, (2008). Preoperative Shower Revisited: Can High Topical Antiseptic Levels Be Achieved on the Skin Surface Before Surgical Admission? Journal of the American College Surgery, 207,233-239.

Edwards P S, Lipp A, Holmes A (2004). Preoperative skin antiseptics for preventing surgical wound infections after clean surgery (Review). The Cochrane Library, 2006. Issue 3

Emori T E, Gaynes R P ( 1993). An overview of nosocomial infections, including the role of the microbiology laboratory. Clinical Microbiology Reviews, 6(4), 428-442-

Flemming K (1998). Asking answerable questions. Evidence-based Nursing, 1(2), 36-37.

Garibaldi R A (1987). Prevention of intraoperative wound contamination with chlorhexidine shower and scrub. Journal of Hospital Infection, Apr; 11 Suppl B, 5-9.

Gravel D, Taylor G, Ofner M, Johnston L, Loeb M, Roth V R, Stegenga J, Bryce E, The Canadian Nosocomial Infection Surveillance Program, Matlow A. (2007). Point prevalence survey for healthcare-associated infections within Canadian adult acute-care hospitals. Journal of hospital infection 66, 243-248.

Harbarth S, Ruef C, Francoli P, Widmwr A, Pittet D (1999). Nosocomial infections in Swiss university hospitals: a multicentre survey and review of the published experience. Schweizerische Medizinische Wochenschrift, 129, 1521-1528. 
Hayek L J, Emerson J M, Gardner A M (1987). A placebo-controlled trial of the effekt of two preoperative baths or showers with chlorhexidine detergent om postoperative wound infection rates. Journal of Hospital Infection, 10(2), 165-172

Kaiser A B, Kernodle D S, Barg N L, Petracek M R (1988). Influence of preoperative showers on staphylococcal skin colonization: A comparative trial of antiseptic skin cleansers. The Annals of Thoracic Surgery, 45(1), 35-38.

La Force F M (1993). The control of infections in hospitals: 1750 to 1950 in prevention and control of nosocomial infections (ed: Wenzel). 2:ed, Willimas and Wilkins.

Lidwell O M (1987). Joseph Lister and infection from the air. Epidemiology and Infection, 99, 569-578.

Lilly H A, Lowbury E J, Wilkins M D (1979). Limits to progressive reduction of resident skin bacteria by desinfection. Journal of Clinical Pathology; 32(4), 382-385

Lindholm, C. (2003). Sår 2:a uppl. Lund: Studentlitteratur

Lowbury E J, Lilly H A, Ayliffe G A (1974). Preopereative disinfection of surgeon's hands: use of alcoholic solutions and effects of gloves on skin flora. British Medical Journal, 4(5941), 369-372.

Mangram A J, Horan T C, Pearson M L, Silver L C, Jarvis W R (1999) The Hospital Infection Control Practice Advisory Committee, Guidelines for the prevention of surgical site infections. American Journal of Infection Control 27(2), 97-132.

Newsom S W, Rowland C (1988). Studies on perioperative skin flora. Journal of hospital infections, 11, suppl B: 21-26 
Nielsen M L, Raahave D, Stage J G and justesen T. (1975). Anaerobic and aerobic skin bacteria before and after skin-disinfection with chlorhexidine: An experimental study in volunteers. Journal of Clinical Pathology, 28, 793-797.

Paulson D S (1993). Efficiacy evaluation of a 4\% chlorhexidine gluconate as a full-body shower wash. American Journal of Infection Control, 21(4), 205-209

Rotter M L, Larsen S O,Cooke E M, Dankert J, Daschner F, Greco D, Grönroos P, Jepsen O B, Lystad A, Nyström B (1988). A comparison of the effects of preperative whole-body bathing with detergent alone and with detergent containing chlor-hexidine gluconate on the frequency of wound infections after clean surgery. The European Working Party on Control of Hospital Infections. Journal of Hospital Infection, 11(4), 310-320.

SBU (1993). Litterature Searching and Evidence Interpretation for Assessing Health Care Practices. Stockholm: Statens beredning för medicinsk utvärdering

\section{SBU: http://www.sbu.se/sv/Evidensbaserad-vard/Faktaruta-1-Studiekvalitet-och-} evidensstyrka/ [2009-11-22]

Segal C G (2006). Infection control: Start with skin. Nursing Management Apr; 37 (4), 46-52

Selwyn S, Ellis H. (1972). Skin bacteria and skin disinfection reconsidered.Br Med $\mathrm{J} ; 1(793): 136-140$.

Socialstyrelsen (2006). Att förebygga vårdrelaterade infektioner. Ett kunskapsunderlag. Stockholm: Socialstyrelsen 
Tammelin A, Klotz F, Hambraeus A, Stahle E, Ransjö U. (2003). Nasal and hand carriage of Staphylococcus aureus in staff at a Department for Thoracic and Cardiovascular Surgery: Endogenous or exogenous source? Infection Control and Hospital Epidemiology; 24(9):686689.

Webster J, Osborne S (2009). Preoperative bathing or showering with skin antiseptics to prevent surgical site infection (Review). The Cochrane Library, 2009. Issue 4

Wihlborg O (1987). The effect of washing with chlorhexidine soap on wound infection rate in general surgery. A controlled clinical study. Annales Chirurgiae et Gynaecoloiae, 76(5), 263265.

Willman A, Stoltz P (2006). Evidensbaserad omvårdnad: en bro mellan forskning och klinisk verksamhet. Lund:Studentlitteratur

Zandi I (2000). Preparing liposuction patients at home preoperatively by scrub-showering. Plastic and Reconstructive Surgery, 106, (1), 219-220 
